\title{
Erratum to: Telerobotic anterior translocation of the ulnar nerve
}

\author{
Jose Carlos Garcia Jr • Gustavo Mantovani • \\ Stephanie Gouzou • Philippe Liverneaux
}

Published online: 24 May 2011

(C) Springer-Verlag London Ltd 2011

\section{Erratum to: J Robotic Surg}

DOI 10.1007/s11701-010-0226-7

Unfortunately due to a typographical error one co-author's name was spelt incorrectly.

The correct spelling of the author's name is stated below:

Philippe Liverneaux

The online version of the original article can be found under doi:10.1007/s11701-010-0226-7.

\section{J. C. Garcia Jr $(\bowtie)$}

Sports Medicine Division/Shoulder and Elbow Surgery Division, Pontifical Catholic University of Campinas Sao Paulo-Brazil, Rua Indiana 670, Brooklin, Sao Paulo, SP 04562-001, Brazil e-mail: josecarlos@especialistadoombro.com.br

G. Mantovani

Sports Medicine Division/Elbow Surgery division, Pontifical Catholic University of Campinas,

Rua Indiana 670, Brooklin, Sao Paulo, SP 04562-001, Brazil

e-mail: mantaray2@uol.com.br

S. Gouzou · P. Liverneaux

Strasbourg University, Hôpitaux Universitaires 1,

place de l'Hôpital, 67091 Strasbourg Cedex, France

e-mail: p.liverneaux@wanadoo.fr;

philippe.liverneaux@chru-strasbourg.fr 